Nathalie Gouleme ${ }^{1}$ Isabelle Scheid ${ }^{2,3}$ Hugo Peyre ${ }^{2,3}$,

Magali Seassau4,

Anna Maruani ${ }^{2,3}$, Julia Clarke ${ }^{2,3}$,

Richard Delorme ${ }^{2,3}$

Maria Pia Bucci'

'UMR 1141 Inserm - Université Paris Diderot. Robert Debré Hospital, 48 Boulevard Sérurier, 75019 Paris, France

${ }^{2}$ Child and Adolescent Psychiatry Department, Robert Debré Hospital, Paris, France

${ }^{3}$ High Functioning Autism Expert Centre, Fondamental Foundation, Paris, France

${ }^{4} e(y e) B R A I N, 1$ bis rue Jean le Galleu, 94200 Ivry-sur-Seine, France

Received 27 June 2017 accepted 25 October 2017

\section{POSTURAL CONTROL AND EMOTION IN CHILDREN WITH AUTISM SPECTRUM DISORDERS}

\section{Abstract}

Autism Spectrum Disorders subjects (ASD) are well known to have deficits in social interaction. We recorded simultaneously eye movements and postural sway during exploration of emotional faces in children with ASD and typically developing children (TD). We analyzed several postural and ocular parameters. The results showed that all postural parameters were significantly greater in children with ASD; ASD made significantly fewer saccades and had shorter fixation time than TD, particularly in the eyes, and especially for unpleasant emotions. These results suggest that poor postural control of ASD and their impaired visual strategies could be due to a lack of interest in social cognition, causing a delay in the development of the cortical areas, and thus could have an effect on their postural control.

\section{Keywords}

Autistic Spectrum Disorders $\bullet$ Childrens $•$ Dual tasks $•$ Emotional faces $\bullet$ Postural controls $•$ Eye movements

\section{Introduction}

Autism Spectrum Disorders (ASD) are neurodevelopmental disorders characterized by deficits in social interaction and communication associated with repetitive, stereotyped behaviors (DSM-5). Several studies have shown impaired postural control abilities among ASD participants. Preliminary studies compared postural sway in children with ASD to those of adults with vestibular disorders and those of control subjects [1]. They showed that postural stability in children with ASD was poor, as compared to control, marked by difficulties adapting their postural stability to environmental conditions. Children with ASD showed a significantly larger surface area of the center of pressure (CoP), specifically in postural conditions where afferent sensory information was disturbed [2]. The authors suggested that children with ASD may have some degree of dysfunction in the brain pathways involved in postural control. Using dynamic posturography, Minshew et al., (2004) [3] explored postural ability on a large sample of participants with ASD aged from 5 to 52 years matched with TD volunteers, and they reported that poor postural control in ASD patients was not compensated during development and persisted in adulthood, at least until 52 years. Reed and McCarthy (2012) [4] compared postural control during a visual task with and without an additional auditory task. They showed that children with ASD were more unstable than controls when they had to integrate both visual and auditory inputs (dual task). In another study by Travers et al., (2013) [5], postural ability was tested on a Wii balance board in several conditions: eyes open, eyes closed, on one or two legs, in 26 ASD adolescents and adults and age-IQ (Intellectual Quotient) matched TD. These authors found worse postural control in ASD with respect to TD on one leg only but not on two legs, thus suggesting that a difficult postural condition could discriminate ASD subjects. Recently, Doumas et al., 2016 [6], compared postural control in young ASD adults with sensory inputs (visual and proprioceptive, separately or simultaneously) with two levels of difficulty. The authors reported worse postural control in ASD when both sensory inputs - visual and proprioceptive - were perturbed. However, they also described that ASD showed a greater postural sway than TD in the lowest level of difficulty condition, thus suggesting a hyper-reactivity in ASD. All these findings are in line with our previous study reporting poor postural stability in ASD children when sensory inputs (visual and proprioceptive) are not available or disturbed, suggesting poor adaptive mechanisms able to compensate environmental changes and maintain postural stability in ASD patients [7]. Recall that in everyday life, we have to assure balance control simultaneously with another task, and attention request is shared between these two tasks. A secondary cognitive task influences the postural stability by increasing or decreasing postural sway depending on the attentional cost involved in the second task. A study recorded the postural sway in children with ASD during a visual searching task (counting the number of specific geometrical forms) and an auditory task (remembering and repeating the numbers they were told) [8]. They showed that postural stability was significantly decreased in children with ASD during these dual tasks,

*E-mail:gouleme.nathalie@gmail.com

(c))BY-NC-ND $\odot 2017$ Nathalie Gouleme et al., published by De Gruyter Open.

This work is licensed under the Creative Commons Attribution-NonCommercial-NoDerivs 3.0 License. 
particularly when the secondary task was visual. In contrast, controls failed to show any postural difference between the tasks. Another study by Stins et al., 2015 [9] compared postural control using both a Wii Balance Board: in eyes closed condition and during a dual cognitive task (word memorization); and Movement Assessment Battery for Children (MABC-2) in ASD children and age- and gender-matched TD. Their findings reported that ASD children had a significantly greater postural sway (more precisely in the medio-lateral direction) than TD in the eyes closed condition only, suggesting that visual input could be more important than attention for obtaining postural stability.

In the present study, children had to free view emotional faces. Although it is still controversial, processing emotional faces is poor in children with ASD [10-19]. Klin et al., (1999) [10], demonstrated that ASD children's deficiency in emotional face processing was not correlated with their cognitive ability. A stereotypical visual pattern (involving exploration of eyes and mouth) was described in TD children during emotional face processing, but ASD children showed a more erratic visual pattern, without exploration of internal features such as eyes and mouth [11]. Golarai et al., (2006) [12], using neuroimaging, described a specific neural network in emotional face processing. These areas are: Superior Temporal Sulcus, Fusiform Face Area and amygdala, respectively involved in gaze processing, face identification and emotion recognition. Moreover, a review by Sasson, (2006) [13], concluded that subjects with ASD showed a deficit in face processing: low level of social interest and innate impairment most likely due to a deficiency in the neural network. Another review by Behrmann et al., (2006) [14], reported that face processing impairment could be due to poor social interaction as well as visual deficit. A study by Boraston et al., (2007) [15], explored eye movements of ASD patients while looking at emotional faces. These authors reported that ASD patients had difficulties in making eye contact and discriminating emotions, specifically for negative emotions. ASD is often associated with deficits in the perception of facial expressions, but the cause of such impairment still remains unclear [15]. For instance, several studies reported specific difficulties for ASD subjects to perceive unpleasant emotions only [16-17], while another study, Meaux et al., (2010) [18], described that ASD children were more efficient than TD children in local processing to discriminate isolated emotions, when looking at several emotional faces: happy, angry, scared, disgusted, sad and surprised. Nevertheless, performance decreased when global processing was required, in line with a lack of central cohesion. Indeed, as suggested by these authors, ASD children show poorer global understanding of their environment than TD children [18]. Recently, a metaanalysis on studies using eye tracking (ET) and electroencephalography (EEG) described that ASD subjects had an impairment in emotional face processing due to a specific visual strategy (found by ET) involving an altered brain function (showed by EEG) - Black et al., (2017) [19].

Free viewing involves various kinds of eye movements such as saccades, fixations, smooth pursuit, vergence, etc. Various studies have shown that eye movements can have an impact on postural sway. Indeed, in adults, several studies described a decrease of postura sway during eye movements (saccades and smooth pursuits) [20-21]. Note that the same phenomenon was also observed in children during saccadic movements [22-23]. Nevertheless, another study addressing a dual task [24] reported that ASD children did not have an improvement of their postural stability independently of the visual performance task (pursuit or saccadic movements), thus suggesting that performing a dual task is very much impaired in children with ASD [25]. Interestingly, a study by Jones \& Klin (2013) [26] recorded with an eye tracker the eye movements of newborn children (from two to six months) during emotional face exploration. They reported that children who later developed ASD did not have a specific visual pattern as that observed in normal children. Thus, this specific different visual strategy developing later in life could be relevant for early therapeutic intervention in ASD children.

The purpose of this study was to record simultaneously postural body sway (with surface, length and mean velocity of the CoP) and eye movements (number of saccades and fixation time) during the exploration of emotional faces among a group of children with ASD and age-, sex- and IQ-matched controls. It is hypothesized that the difficulties consistently observed in children with ASD in processing the emotions on faces could affect the control of their postural body performances. Moreover, as previously mentioned above, eye movements have an impact on body sway. Consequently, in this dual task we can make the hypothesis that free viewing of emotional faces could involve a change in postural control depending on the visual strategies used by ASD children.

\section{Materials and methods}

\section{Participants}

Thirty children with ASD (mean age: $12.1 \pm 2.9$ years) and thirty age-, sex- and IQ-matched typically developing children were included in the study. All children enrolled in the study had a neurological exam in the normal range and were psychotropic drug-naïve. None had a personal history of sensory deficit (in any of the five senses). The diagnosis of ASD was based upon evaluation data from the Autism Diagnostic Interview-Revised (ADI-R) [27], the Autism Diagnostic Observation Schedule (ADOS) [28] and expert clinical judgment based on DSM-5 criteria. The cognitive abilities of the participants were also assessed using the Wechsler Intelligence Scale for children (WISC IV), the Movement Assessment Battery for Children (M-ABC) [29] and the Beery-Buktenica Developmental Test of Visual-Motor Integration (VMI). All these tests allow a precise evaluation of children with autistic spectrum disorder. They were enrolled at the Expert Centre for High Functioning ASD (supervised by $\mathrm{Pr}$. Richard Delorme) at the Robert Debré pediatric hospital in Paris.

These thirty ASD children were matched with thirty typically developing children (TD) in age, sex and IQ to ensure good reproducibility. TD children displayed no personal history of neurological or psychiatric disorders. IQ was estimated on two subtests of the WISC-IV, one assessing their verbal capabilities (Similarities test) and one assessing their performance 
capabilities (Matrix Reasoning test). The scores at these two tests were not significantly different between the two groups $\left(F_{(1,74)}=\right.$ $1.02, p=0.3$ and $F_{(1,74)}=1.45, p=0.10$ for the similarity and matrix test, respectively). The clinical characteristics of children with ASD and TD are summarized in Table 1. TD recruitment was based on voluntary participation. Note that no participant (ASD or TD subject ) dropped out during our study, and based on previous work it is assumed that thirty children were enough considering their age range [30-31].

The investigation followed the principles of the Declaration of Helsinki and was approved by our Institutional Human Experimentation Committee (Comité de Protection des Personnes CPP lle de France, Hôpital SaintAntoine). Written consents were obtained from the children's parents after the experimental procedure was explained to them.

\section{Visual stimuli}

The stimuli were presented on a 22-inch PC screen with a resolution of $1920 \times 1080$ and a refresh rate of $60 \mathrm{~Hz}$. Five Ekman's emotion pictures (one happy face and three faces expressing sadness, fear and angry, and a neutral face as a control condition) were randomly presented to the children during the postural measures $(25.6 \mathrm{sec})$. All the $11.8^{\circ}$ $x 15.2^{\circ}$ pictures are shown in Figure 1 [32]. The emotional faces were presented once for 25.6 seconds each (that is the postural recording time). After a rest of a few seconds, to reduce any possible fatigue effect, another emotional face was presented (see [25] for more details). All emotional faces were divided according to their regions of interest (ROIs): the screen (without any emotional face), eyes $\left(8.4^{\circ} \times 8^{\circ}\right)$, nose $\left(4.6^{\circ} \times 2^{\circ}\right)$ and mouth $\left(5^{\circ} \times 4.5^{\circ}\right)$ (see Figure 1). This specific rational division of emotional faces allowed analysis of eye movement parameters with better reproducibility (using Meye Analaysis software). Identical methods have been used in a previous study [31]. Previously, each emotional face was divided into two additional ROIs: the top of the face (including eyes, forehead, part of hair, top of nose, ears and cheeks), and the bottom of the face (including mouth, chin, bottom of the

Table 1: Clinical characteristics of children with ASD and typical development children (TD).

\begin{tabular}{lcc}
\hline & $\begin{array}{c}\text { Children with ASD } \\
\mathbf{N}=\mathbf{3 0}\end{array}$ & $\begin{array}{c}\text { Children with typical } \\
\text { development } \\
\mathbf{N}=\mathbf{3 0}\end{array}$ \\
\hline Age (years), mean (SD) & $12.1(2.9)$ & $11.08(0.5)$ \\
Gender (male), \% & 86.7 & 86.7
\end{tabular}

Diagnosis of ASD

AutismDiagnosticlnterview-Revised(ADI-R) scores

Communication, mean(SD)

$11.8(4.4)$

Stereotyped Patterns of Behaviors, mean (SD)

$4.9(2.3$

Autism Diagnostic Observation Schedule (ADOS) scores

$\begin{array}{ll}\text { Social Reciprocallnteraction, mean (SD) } & 8.0(3.3) \\ \text { Communication, mean(SD) } & 4.0(1.6)\end{array}$

Cognitive assessment

Wechsler scale (WISC-IV) scores

Verbal Comprehension, mean (SD)

Perceptual Reasoning, mean (SD)

$91.2(23.1)$

Working Memory, mean (SD)

$87.5(20.1)$

Processing Speed, mean (SD)

$86.2(19.9)$

Similarities, mean (SD)

$10.1(0.9)$

$10.7(0.6)$

Matrix Reasoning, mean (SD)

$8.9(0.7)$

$9.2(0.5)$

Movement Assessment Battery for Children

Total impairment score, mean (SD)

$15.0(7.3)$

Visual-Motor Integration test

Standardized score (SD = 10), mean (SD)

$6.0(3.1)$

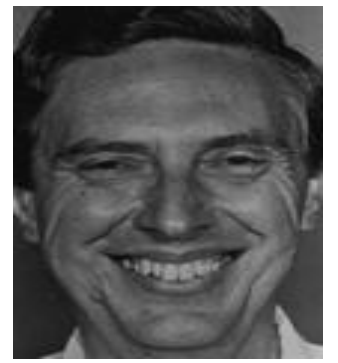

A

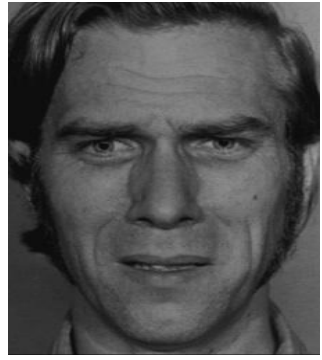

B

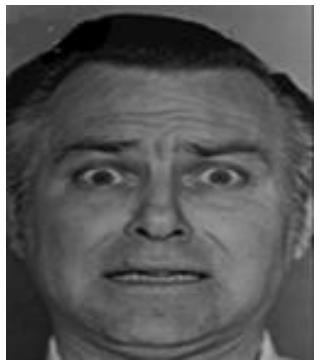

C

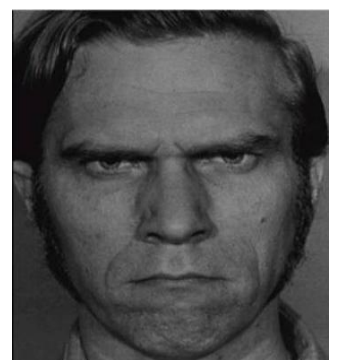

$\mathrm{D}$

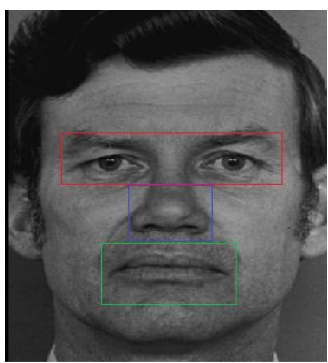

E

Figure 1: Five emotional faces: happy (A), sad (B), fear (C), angry (D) and neutral (E). Regions of interests (ROIs) are shown in the neutral face (E). 
nose, cheeks and ears). However, no significant difference was observed in any with these two additional ROls both for the number of saccades, fixation time, independently of emotional face.

\section{Postural recordings}

A platform of two dynamometric clogs (standards by Association Française de Posturologie 1985, 1986, produced by TechnoConcept ${ }^{\circ}$, Céreste, France) was used to measure the subject's postural stability while he or she was looking at an emotional face. Surface area, the mean velocity and the length of the CoP during the presentation time, i.e. 25.6 seconds were analyzed. These postural parameters allow efficient measurement of CoP spatial variability [33]. The surface of the CoP corresponds to an ellipse with $90 \%$ of CoP excursions. The length of the CoP is the path of the center of pressure. Software allows two components of length of postural sway: in medio-lateral and antero-posterior. Taken into account were only length in anteroposterior direction due to its more ecological used direction for activities in everyday life, as previously analyzed in several studies [31; 34-38]. The mean velocity of the CoP is the mean velocity of the CoP displacements over the sampled period, that is, the sum of the displacement scalars over the sampling period divided by the sampling time. It represents a good index of the amount of neuromuscular activity required to regulate postural control [39]. Postural sway of each human body shows its own surface area and can change depending on conditions or individual factors. Moreover, for a same surface area obtained, the postural sway can change quickly or very slowly over time leading to different mean velocities of CoP displacement.

\section{Eye movement recordings}

Eye movements were recorded during the postural measures with the Mobile Eyebrain Tracker (Mobile EBT ${ }^{\circ}$, e(ye)BRAIN), an eyetracking device $C E$ marked for medical purposes. The Mobile EBT" benefits from a high frequency camera that can record both the horizontal and vertical eye positions independently and simultaneously for each eye. Recording frequency was set up to $300 \mathrm{~Hz}$. The precision of this system is $0.5^{\circ}$. The recording system does not obstruct the visual field, and the calibrated zone covers a horizontal visual angle of $\pm 22^{\circ}$. Number of saccades and the time spent on each $\mathrm{ROI}$ were analyzed with the MeyeAnalysis software, during the first five seconds and over the total time of visual exploration $(25.6 \mathrm{sec}$ ); recall however that several studies suggested that the processing of emotional faces occurs during the first seconds of exploration [40-41]. Note that the software allows measurement of saccadic eye movements between two fixations in a specific ROI.

\section{Experimental procedure}

Each participant was assessed in a dark room to reduce any visual distractor from the specific task of exploration. Children were standing on the platform, in front of the screen located $60 \mathrm{~cm}$ away from him/her. Postural and oculomotor measurements were performed while the subject explored a face. A calibration for eye movement recordings was conducted before the stimulus presentation. During the calibration procedure, the children were asked to fixate on a grid of 13 points (diameter 0.5 deg) mapping the screen. Each calibration point required a fixation time of $250 \mathrm{~ms}$ to be validated. After the calibration procedure, the children were invited to look at an emotional face on the screen in front of them during 25.6 seconds. The children were asked to stand up on the platform, without any voluntary movements, their arms along side their bodies. One easy and short instruction was given: "Look at the emotional face without moving your body." Each postural and oculomotor recording test was followed by a rest lasting a few seconds, while a new emotional face was randomly selected. Each emotional face was presented, randomly, one single time, during 25.6 seconds, thus postural control and eye movements were recorded one single time for each emotional face (happy, sad, fear, angry and neutral). Due to the low weight of this specific eye tracker $(350 \mathrm{~g})$ and the short recording time of our experimental procedure, even the youngest subject in our sample (8 years old) cooperated well, without dropping out.

\section{Statistical analysis}

Analysis of variance (ANOVA) was determined with mixed-design multivariate using the two groups and the five emotional faces - groups (2), emotional faces (5) - for postural parameters (surface, length and mean velocity of the CoP). Then another ANOVA was performed with eye movements (number of saccades and fixation time during the first five seconds of exploration and during the total time of exploration, i.e. 25.6 seconds) for the groups (2), emotional faces (5) and ROIs (4).

Post hoc comparisons were made with the Fischer's least significant differences test (LSD). The effect of a factor was considered as significant when the $p$-value was below 0.05 .

\section{Results}

Firstly, no significant correlation between clinical evaluations and postural or eye movement parameters.

\section{Surface area of $\mathrm{COP}$}

Figure $2 \mathrm{~A}$ shows the surface area with mean and standard error of the CoP $\left(\mathrm{mm}^{2}\right)$ for both ASD and TD children during the exploration of faces displaying the four emotions (happy, sad, fear, angry) and those that were neutral. The analysis of variance (ANOVA) showed an effect of group $\left(F_{(1,58)}=15.58, p<0.0001\right)$. The children with ASD had a significantly greater surface of the CoP than TD. There was a significant effect of emotion $\left(F_{(4,232)}=3.21, p<0.01\right)$. The LSD test showed that independently of the group the children belonged to, the surface area of the CoP was significantly greater in front of a happy face than in front of a neutral or angry face (all $p<0.02$ ). However, these results showed no significant interaction between groups and emotions.

\section{Length of the COP}

Figure $2 \mathrm{~B}$ shows the length with mean and standard error of the CoP $(\mathrm{mm})$ for both ASD and TD children during the exploration of the faces showing emotions. The analysis of variance (ANOVA) showed a significant group effect $\left(F_{(1.58)}=4.85, p<0.03\right)$ : the CoP was significantly longer in ASD than in TD children. Also, there was a significant effect of emotion 
$\left(F_{(4,232)}=3.81, p<0.006\right)$. The LSD test showed that independently of the group, the length of the CoP was significantly greater in front of a happy face than in front of neutral, fear or angry faces (all $p<0.02$ ). Moreover, there was a significant interaction between group and emotion $\left(F_{(4,232)}=2.99, \mathrm{p}<0.02\right)$. The LSD test showed that ASD children facing happy and sad emotional faces had a significantly greater length of the CoP than TD children (all $p<0.01$ ).

\section{Mean velocity of the CoP}

Figure $2 \mathrm{C}$ shows the mean velocity with means and standard error of the CoP $(\mathrm{mm} / \mathrm{s})$ for both ASD and TD children for the five emotional faces. The analysis of variance (ANOVA) showed a significant group effect $\left(F_{(1,58)}=6.11, p<0.02\right)$. Children with ASD had a significantly greater mean velocity of the CoP than that of the TD. Moreover, there was a significant effect of emotion $\left(F_{(4,232)}=2.67, p<0.03\right)$. Indeed, LSD test showed that independently of the groups, the mean velocity of the CoP was significantly greater in front of a happy face than in front of a face showing fear, angry and neutral emotions (all $\mathrm{p}<0.02$ ). Moreover, there was a significant interaction between group and emotion $\left(\mathrm{F}_{(4,232)}\right.$ $=2.76, p<0.03)$. LSD test showed that the mean velocity of the CoP for ASD children was significantly greater in front of happy and sad faces than for the TD (all $p<0.02$ ).

\section{Number of saccades in ROls}

Figure 3 shows the number of saccades in ROls (screen, eyes, nose and mouth) with mean and standard error for both ASD and TD children looking at the emotional faces during the first five seconds of exploration (A) and during the total time of exploration (25.6 seconds) (B). Concerning the first five seconds of exploration, analysis of variance (ANOVA) showed a significant group effect $\left(F_{(1,58)}=8.63\right.$, $\mathrm{p}<0.006$ ): ASD children had a significantly smaller number of saccades than TD children. Interestingly, there were three interactions: between groups and ROls $\left(F_{(3,174)}=3.36\right.$, $\mathrm{p}<0.02)$, between emotions and ROls $\left(\mathrm{F}_{(12,696)}=\right.$ $1.48, \mathrm{p}<0.001)$, and between groups, emotions and ROls $\left(F_{(12,696)}=1.98, p<0.02\right)$. The LSD test reported that ASD children had a significantly smaller number of saccades in the eyes ROI

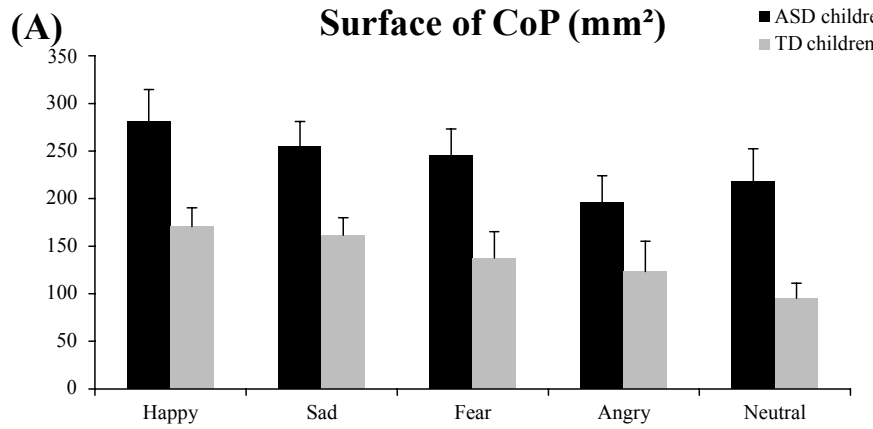

(B) Length of CoP (mm)

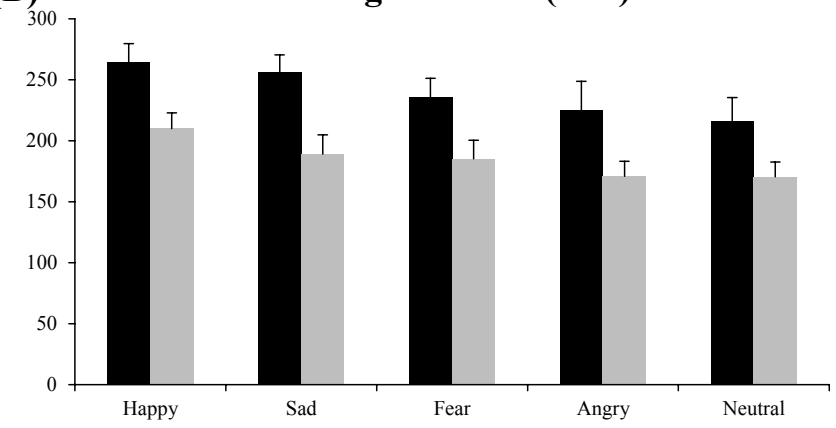

(C)

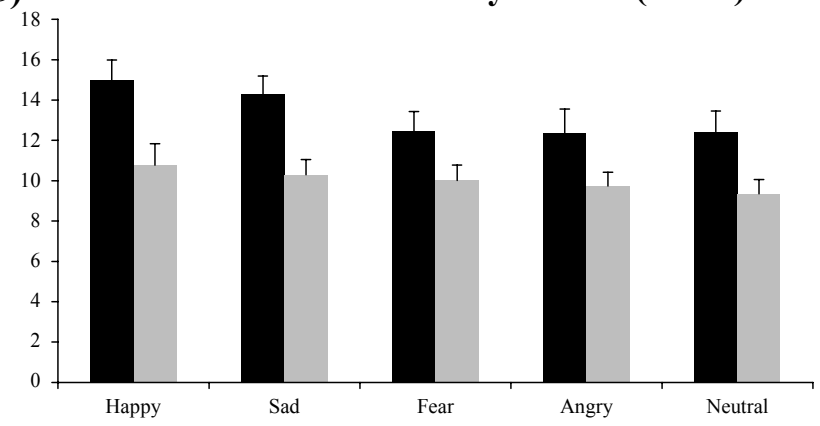

Figure 2: Surface area $\left(\mathrm{mm}^{2}\right)(A)$, length $(\mathrm{mm})(B)$ and mean velocity $(\mathrm{mm} / \mathrm{s})(C)$ values of the CoP for both ASD and TD for the five emotional faces (happy, sad, fear, angry, and neutral) during postural recordings. Vertical bars indicate standard error.

than TD $(p<0.001)$. Also, independently of the groups of children, the LSD test showed that the number of saccades in the eyes ROI for unpleasant faces (sad, fear and angry) was significantly greater than that for neutral and happy emotional faces (all $p<0.01$ ). Finally, the LSD test showed that the number of saccades in the eyes ROI for unpleasant faces in ASD children was significantly smaller than that of TD children (all $p<0.04$ ).

Concerning the total time of exploration: 25.6 seconds, analysis of variance (ANOVA) showed a significant group effect $\left(\mathrm{F}_{(1,58)}=\right.$ 1.31, $\mathrm{p}<0.001$ ): ASD children had significantly smaller number of saccades than TD children. Moreover, there was a significant effect of ROI $\left(F_{(3,174)}=22.44, p<0.001\right)$. LSD test showed that independently of the group of children, the number of saccades was significantly greater in eyes ROI than in nose and mouth ROls (all $p<$ 0.001). Also, the number of saccades in screen $\mathrm{ROI}$ was significantly greater than in nose and mouth ROls (all $p<0.001$ ). Finally, ANOVA showed a significant interaction between 


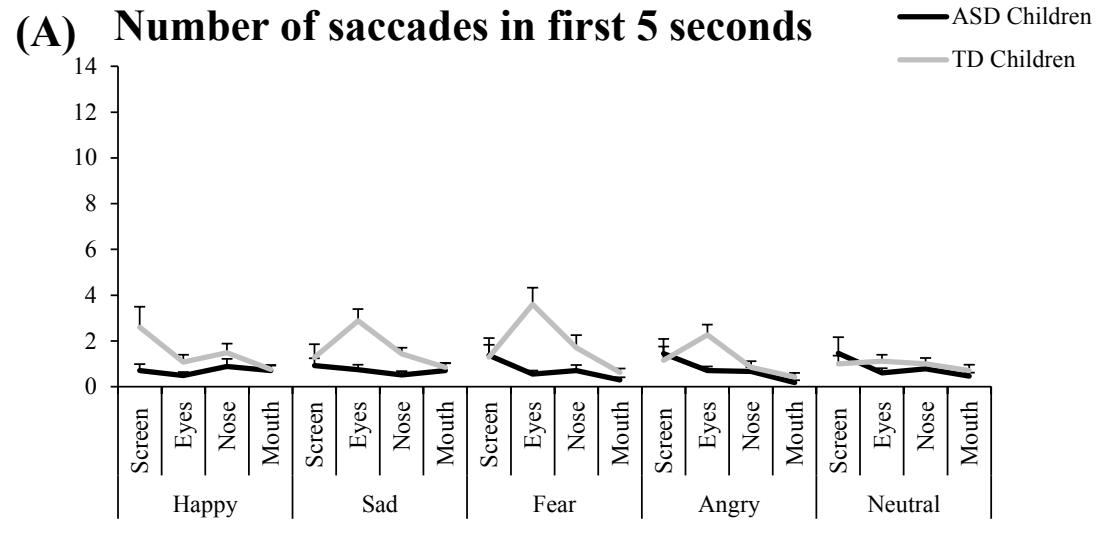

(B) Number of saccades during 25,6 seconds

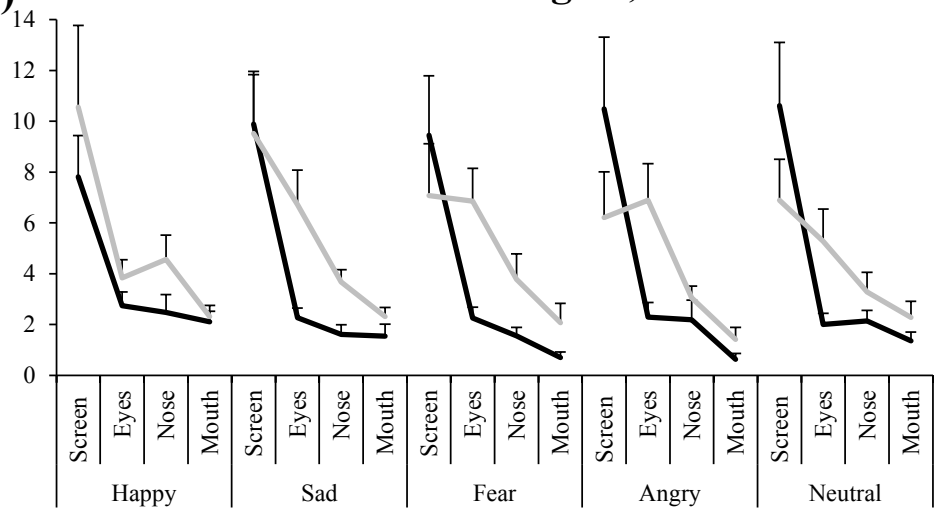

Figure 3: Number of saccades in ROIs (screen, eyes, nose and mouth) for both ASD and TD children for the five emotional faces (happy, sad, fear, angry, and neutral) during the first five seconds of exploration (A) and during the total time of exploration: 25.6 seconds (B). Vertical bars indicate standard error.

group and $\mathrm{ROI}\left(\mathrm{F}_{(3,174)}=2.24, \mathrm{p}<0.003\right)$ : indeed, LSD test reported that the number of saccades in eyes ROI in ASD children was significantly smaller with respect to that of TD children (all $\mathrm{p}<0.001$ ).

\section{Fixation time in ROIs}

Figure 4 shows the fixation time in ROIs (screen, eyes, nose and mouth) with mean and standard error for both ASD and TD children during the first five seconds of exploration of the faces (A) and during the total time of exploration (25.6 seconds) (B). Indeed, the processing of emotional face occurs very early in beginning of exploration.

Concerning the first five seconds of exploration, analysis of variance (ANOVA) showed a significant group effect $\left(F_{(1,58)}=4.06\right.$ $\mathrm{p}<0.001)$ : the fixation time was significantly shorter in the children with ASD than in the TD. Furthermore, there was a significant interaction between group emotion and $\mathrm{ROI}\left(\mathrm{F}_{12,696)}=1.12\right.$, $\mathrm{p}<0.003)$. The children with ASD explored significantly less the eyes $\mathrm{ROI}$ for unpleasant emotional faces than the TD (all $p<0.004$ ).

Concerning the total time of exploration (25.6 seconds), analysis of variance (ANOVA) showed a significant group effect $\left(\mathrm{F}_{(1,58)}=0.62\right.$ $p<0.04)$ : the fixation time in ASD children was significantly shorter than that of TD children. Moreover, there was a significant effect of ROI $\left(F_{(6,96)}=13.49, p<0.001\right)$. LSD test showed that independently of the group of children, the time of fixation was significantly greater in the eyes ROI than in the nose and mouth ROIs (all $\mathrm{p}<0.001$ ).

\section{Discussion}

The aim of this study was to explore simultaneously the postural body sway and eye movements of a group of children with ASD and age-, sex- and IQ-matched TD while they explored emotional faces. As first hypothesized, it was observed that the postural control of children with ASD is more strongly impacted during the exploration of emotional faces than that of TD children.

As previously reported by various groups, several postural parameters (surface, length and mean velocity of the $(0 P$ ) were significantly affected in children with ASD compared to TD children [1-3; 7, 31]. Children with ASD displayed poorer abilities to maintain their postural control, whatever the condition of the exploration (simple or dual tasks).

Even if the correct recognition of the emotional face in our groups of children, was not verified children with ASD used different visual strategies to explore emotional faces. It was observed that during this dual task, children with ASD made fewer saccades while exploring emotional faces, particularly for the eyes ROI, and had a shorter fixation time than TD children. These results confirmed the vast literature showing that children with ASD spent significantly less time in the eyes region, which is the most relevant area for processing emotions [45-47]. Moreover, children with ASD made significantly fewer saccades and had a shorter fixation in the eyes region when seeing unpleasant emotional faces (sad, fear and angry), emotions that are known to be more difficult to apprehend for ASD children. The cortical area involved in processing unpleasant faces seems distinct from that involved in pleasant emotional face recognition. Indeed, processing an unpleasant emotion matures earlier with respect to those involved in pleasant emotions [47]. Children with ASD may experience a delay in the maturity of the neural networks involved in unpleasant emotional face recognition. Importantly, in ASD children, this specific visual strategy (with fewer saccades 
and a short fixation time) is maintained during the whole time of exploration.

Nevertheless, our results do not report a significant effect of emotion after the first five seconds of exploration according to several studies [40-42]. Indeed, the emotional processing involves the first saccades and thus takes place at the beginning of the exploration.

Furthermore, it was reported that children with ASD increased postural parameters when facing emotional faces. During this dual task, children with ASD focusing their attention on free viewing of emotional faces increased their postural control instability. The exploration of the emotions for participants with ASD is associated with high attention allocation and thus interferes with their ability to control their posture [48]. The hypothesis could not be made that ASD children have a poor attention resource allocation, in this specific dual task and, thus, less adaptive mechanisms to compensate postural changes in a natural environment. These findings are in line with our recent study [7]. Indeed, we described that ASD children had a poor postural control when sensory inputs (visual and proprioceptive) were not available due to their inability to use compensatory sensory strategy to maintain their postural control. Nevertheless, an interesting study [49] described that postural sway in the mediolateral direction decreased significantly in ASD children with a light touch with finger, independently with eyes closed or open. These results suggest that a supplementary proprioceptive input can help to decrease postural impairment of ASD children.

These current results support those which show that postural stability is significantly decreased in children with ASD during dual tasks, specifically when the secondary task is visual [8]. These authors recorded postural sway in children with ASD during a visual searching task, which was probably more basic in terms of cognitive processing than the exploration of emotional faces. In their study, the particpants had to count the number of specific geometrical forms during visual exploration. Interestingly, the postural sway was significantly greater in ASD children in front of happy and sad emotional faces

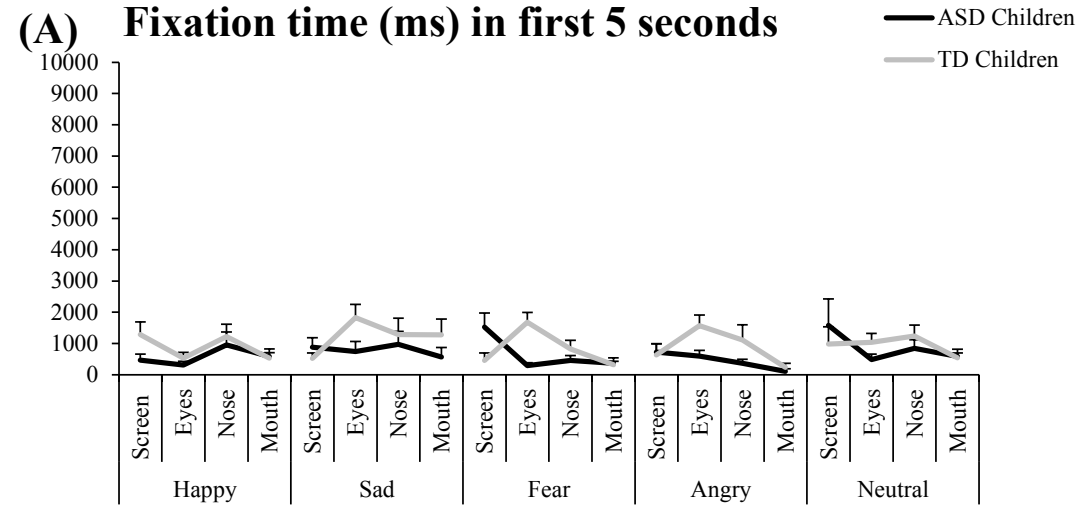

(B) Fixation time (ms) during 25,6 seconds

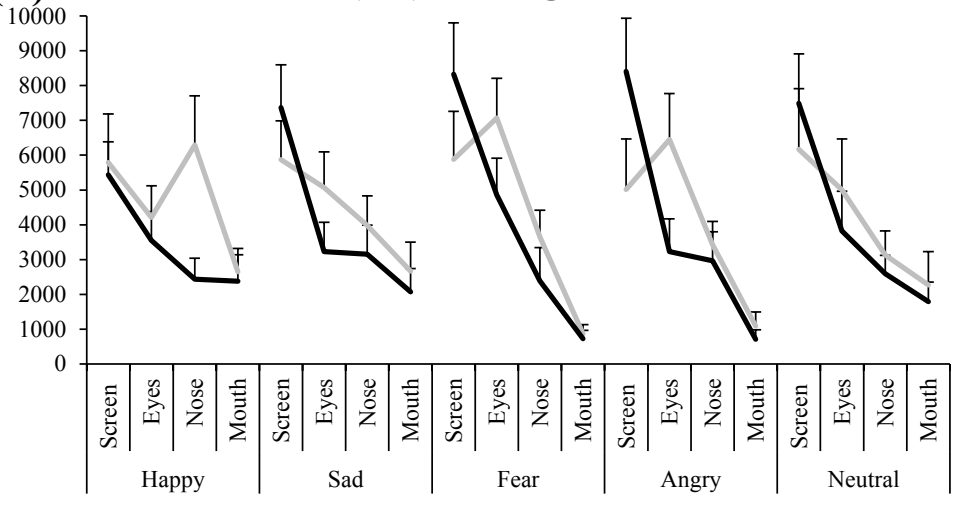

Figure 4: Fixation time in ROls (screen, eyes, nose and mouth) for both ASD and TD children for the five emotional faces (happy, sad, fear, angry, and neutral) during the first five seconds of exploration (A) and during the total time of exploration: 25.6 seconds (B). Vertical bars indicate standard error.

with respect to TD children. We suggested that these two emotional faces are the most frequently encountered in everyday life and could lead to increased difficulties in postural control, as reported in clinical symptoms. It has been reported a significantly greater interest in the mouth region in ASD with respect to TD children [50]. Moreover, happy emotional faces are specific due to the mouth region, which can be considered as a critical factor in facilitating discrimination, as reported in a previous study [42]. Nevertheless, despite the results of our current study, we have not found a significant effect concerning the mouth $\mathrm{ROI}$ in both groups of children tested. Finally, the limit of this study is that the postural abnormality reported in ASD children and specific visual strategies are not correlated with the results of their clinical tests. Further studies on a large sample of ASD children and an analysis on more ocular parameters will be necessary in order to better understand the issue. It could also be interesting to use ecological scenes showing the everyday life of children (school, family, leisure, etc.) in order to explore further their social interaction deficiencies and the impact on their postural capability in their daily lives. Thus, specific remediation could be proposed to such children in order to improve both their visual and postural strategies. Moreover, in this current study, ASD children were not evaluated on a single task; thus, we are not able to confirm the magnitude of the exploration of emotional faces on postural deficiency.

\section{Conclusion}

In the current study we explored the postural control during a dual task of emotional face processing in ASD compared to TD children. 
Children with ASD had both a poorer postural control and deficits in the visual strategy used to explore emotional faces. Moreover, while exploring happy and sad emotional faces, ASD children increased their postural sway.

\section{Ethical approval}

All procedures performed in studies involving human participants were in accordance with the ethical standards of the institutional and/or national research committee and with the 1964 Helsinki declaration and its later amendments or comparable ethical standards. Informed consent was obtained from all individual participants included in the study.

References

[1] Kohen-Raz R., Volkmar F.R., Cohen D.J. Postural control in children with autism, Journal of Autism and Developmental Disorders, 1992, 22(3), 419-432.

[2] Molloy C.A., Dietrich K.N., Bhattacharya A. Postural stability in children with autism spectrum disorder, Journal of Autism Developmental Disorder, 2003, 33(6),643-652.

[3] Minshew N.J., Sung K., Jones B.L., Furman J.M. Underdevelopment of the postural control system in autism, Neurology, 2004, 63(11),20562061.

[4] Reed P., McCarthy J. Cross-modal attention-switching is impaired in autism spectrum disorders, Journal of Autism and Developmental Disorders, 2012, 42(6),947-952.

[5] Travers B.G., Powell P.S., Klinger L.G., Klinger M.R. Motor difficulties in autism spectrum disorder, linking symptom severity and postural stability, Journal of Autism Developmental Disorders, 2013, 43,1568-1583.

[6] Doumas M., McKenna R., Murphy B. Postural deficits in autism spectrum disorder, The role of sensory integration, Journal of Autism Developmental Disorder, 2016, 46,853-861.

[7] Goulème N., Scheid I., Peyre H., Maruani A., Clarke J., Delorme R., Bucci M.P. Spatial and temporal analysis of postural control in children with high functioning Autism Spectrum Disorder, Gait \& Posture, 2017, 40,13-23.

[8] Memari A.H., Ghanouni P., Shayestehfar M., Ziaee V., Moshayedi P. Effects of visual search vs. auditory tasks on postural control in children with autism spectrum disorder, Gait \& Posture, 2014, 39(1),229-234.

[9] Stins J.F., Emck C., de Vries E., Doop S., Beek P.J. Attentional and sensory contributions to postural sway in children with autism spectrum disorders, Gait Posture, 2015, 42,199-203.

[10] Klin A., Sparro S.S., de Bildt A., Cicchetti D.V., Cohen D.J., Volkmar F.R. A Normed Study of Face Recognition in Autism and Related Disorders, Journal of Autism and Developmental Disorders, 1999,29(6).

[11] Pelphrey K.A., Sasson N.J., Reznick J.S., Paul G., Goldman B.D., Piven J. Visual scanning of faces in autism, Journal of autism and developmental disorders, 2002, 32(4),249-261.

[12] Golarai G., Grill-Spector K., Reiss A.L. Autism and the development of face processing, Clinical Neuroscience Research, 2006, 6,145-160.

[13] Sasson N.J. The Development of Face Processing in Autism, Journal of Autism and Developmental Disorders, 2006, 36,(3), doi:10.1007/ s10803-006-0076-3.

[14] Behrmann M., Thomas C., Humphreys K. Seeing it differently, visual Processing in autism, TRENDS in Cognitive Sciences, 2006, 10(6).
[15] Boraston Z., Blakemore S.J. The application of eye-tracking technology in the study of autism, Physiology, 2007, 15,581(3),893898.

[16] Humphreys K., Minshew N., Leonard G.L., Behrmann M. A finegrained analysis of facial expression processing in high-function adults with autism, Neuropsychologia, 2007, 2,45(4),685-695.

[17] De Jong M.C., Van Engelund H., Kemner C. Attentional effects of gaze shifts are influenced by emotion and spatial frequency, but not in autism, Journal of American Academy of Child and Adolescent Psychiatry, 2008, 47(4),443-454.

[18] Meaux E., Gillet P., Bonnet-Brilhault F., Barthélémy C., Batty M. Atypical perception processing and facial emotion disorder in autism, Encephale, 2010, 37(5),371-378.

[19] Black M.H., Chen N.T.M., lyer K.K., Lipp O.V., Bölte S., Falkmer M., Tan T., Girdler S. Mechanisms of facial emotion recognition in autism spectrum disorders, Insihts from eye tracking and electroencephalography, Neuroscience and Biobehavioral reviews, 2007, 8,80,488-515.

[20] Rougier P., Garin M. Performing saccadic eye movements or blinking improves postural control, Motor Control, 2007, 11(3),213-223.

[21] Stoffregen T.A., Bardy B.G., Bonnet C.T., Hove P., Oullier O. Postural sway and the frequency of horizontal eye movements, Motor Control, 2007, 11(1),86-102.

[22] Ajrezo L., Wiener-Vacher S., Bucci M. P. Saccades Improve Postural Control, A Developmental Study in Normal Children, PLoS ONE, 2013, 8(11), doi:org/10.1371/journal.pone.0081066.

[23] Bucci M.P., Seassau M., Larger S., Bui-Quoc E., Gerard C.L. Effect of visual attention on postural control in children with attention-deficit/ hyperactivity disorder, Research Developmental Disabilities, 2014, 35(6), 1292-300.

[24] Bucci M.P., Doyen C., Contenjean Y., Kaye K. The Effect of Performing a Dual Task on Postural Control in Children with Autism, Interantional Scholary Research Neursocience, 2013, doi:10.1155/2013/796174.

[25] Courchesne E., Townsend J., Akshoomoffetal N.A. Impairment in shifting attention in autistic and cerebellar patients, Behavioral Neuroscience, 1994, 108(5),848-865.

[26] Jones W., Klin A. Attention to Eyes is Present But in Decline in 2-6 Month-Olds Later Diagnosed with Autism, Nature, 2013, 19,504(7480), 427-431.

[27] Lord C., Rutter M., Le Couteur A. Autism Diagnostic InterviewRevised, a revised version of a diagnostic interview for caregivers of individuals with possible pervasive developmental disorders, Journal 
of Autism and Developmental Disorders, 1994, 24(5),659-685.

[28] Lord C., Risi S., Lambrecht L., Cook E.H., Leventhal B.L., Di Lavore P.C., Pickles A., Rutter M. The autism diagnostic observation schedulegeneric, a standard measure of social and communication deficits associated with the spectrum of autism, Journal of Autism and Developmental Disorders, 2000, 30(3),205-223.

[29] Henderson S.E., Sugden D.A. The Movement Assessment Battery for Children, San Antonio, TX, The Psychological Corporation, 1992.

[30] Goulème N., Ezane M.D., Wiener-Vacher S., Bucci M.P. Spatial and temporal postural analysis, a developmental study in healthy children, International Journal of Developmental Neuroscience, 2014, 38,169-177.

[31] Goulème N., Seassau M., Bucci M.P. The effect of face exploration on postural control in healthy children, Gait \& Posture, 2015, 12(2),178185.

[32] Ekman P., Friesen W.V. Constants across cultures in the face and emotion, Journal of personality and social psychology, 1971, 17(2), 124-129.

[33] Chiari L., Rocchi L., Capello A. Stabilometric parameters are affected by anthropometry and foot placement, Clinical Biomechanics, 2009, 17(9-10),666-677.

[34] Lions C., Colleville L., Bui-Quoc E., Bucci M.P. Importance of visual inputs quality for postural stability in strabismic children, Neuroscience Letters, 2016, 23,617,127-133.

[35] Lions C., Bui Quoc E., Wiener-Vacher S., Bucci M.P. Postural control in strabismic children, importance of proprioceptive information, Frontiers in Physiology, 2014, 23,5,156.

[36] Lions C; Bui-Quoc E., Bucci M.P. Postural control in strabismic children versus non strabismic age-matched children, Graefes Archive for Clinical and Experimental Ophthalmology, 2013, 251(9),2219-2225.

[37] Bucci M.P., Soufi H., Villeneuve P., Colleville L., Bui-Quoc E., Lions C. Importance of Proprioceptive Information for Postural Control in Children with Strabismus before and after Strabismus Surgery, Frontieres in Systems Neuroscience, 2016, 6,10,67.

[38] Goulème N., Gerard C.L., Bucci M.P. Postural Control in Children with Dyslexia, Effects of Emotional Stimuli in a Dual-Task Environment, Dyslexia, 2017, 23(3),283-295.

[39] Geurts A.C., Nienhuis B., Mulder T.W. Intrasubject variability of selected force-platform parameters in the quantification of postural control, Archives of physical medicine and rehabilitation, 1993, 74(11), 1144-1150.

[40] Krolak-Salmon P., Hénaff M.A., Vighetto A., Bertrand O., Mauguière F. Early amygdala reaction to fear spreading in occipital, temporal, and frontal cortex, a depth electrode ERP study in human, Neuron, 2004, 27,42(4),665-676.

[41] Calvo M.G., Nummenmaa L. Time course of discrimination between emotional facial expressions, The role of visual saliency, Vision Research, 2011, 51(15),1751-1759.

[42] Hot P., Sequeira H. Time course of brain activation elicited by basic emotions, Neuroreport, 2013, 24(16),898-902.

[43] Jansiewicz E.M., Goldberg M.C., Newschaffer C.J., Denckla M.B., Landa R. Mostofsky S.H. Motor signs distinguish children with high functioning autism and Asperger's syndrome from controls, Journal of Autism Developmental Disorders, 2006, 36(5),613-621.

[44] Hood B.M., Willen J.D., Driver J. Adult's eyes trigger shifts of visual attention in human infants. Clinical psychological science, 1998, 9(2),131-134.

[45] Klin A., Jones W., Schultz R., Volkmar F., Cohen D. Visual fixation patterns during viewing of naturalistic social situations as predictors of social competence in individuals with autism, Archives of General Psychiatry, 2002, 59(9), 809-816.

[46] Schyns P.G., Petro L.S., Smith M.L. Dynamics of visual information integration in the brain for categorizing facial expressions, Current Biology, 2007, 17(18), 1580-1585.

[47] Batty M., Taylor M.J. The development of emotional face processing during childhood, Developmental Sciences, 2006, 9(2),207-220.

[48] Gillespie-Smith K., Riby D.M., Hancock P.J., Doherty-Sneddon G. Children with autism spectrum disorder (ASD) attend typically to faces and objects presented within their picture communication systems, Journal of intellectual disability research, 2014, 58(5),459470.

[49] Chen F-C, Tsai C-L. A light fingertip touch reduces postural sway in children with autism spectrum disorders, Gait Posture, 2016, 43,137140.

[50] Rutherford M.D., Clements K.A., Sekuler A. Differences in discrimination of eye and mouth displacement in autism spectrum disorders, Vision Research, 2007, 47,2099-2110. 